\title{
Ictiose arlequim - manejo do nascimento aos 3 anos de idade
}

\author{
Harlequin ichthyosis - management from birth to 3 years of age
}

\author{
Isabelle Sasso Teixeira ${ }^{1}$, Grace Rodrigues ${ }^{1}$, Guilherme Nazar ${ }^{1}$, Izabelle Schermak das Neves ${ }^{1}$, \\ Danielle Arake Zanatta ${ }^{2}$, Vânia Oliveira Carvalho ${ }^{3}$
}

Teixeira IS, Rodrigues G, Nazar G, Neves IS, Zanatta DA, Carvalho VO. Ictiose Arlequim - manejo do nascimento aos 3 anos de idade / Harlequin Ichthyosis - management from birth to 3 years of age. Rev Med (São Paulo). 2020 jul.-ago.;99(4):411-4.

RESUMO: A ictiose arlequim possui uma prevalência de 1/100.000 nascimentos e é frequente o óbito ocorrer no período neonatal. Objetivo: descrever um caso em que o tratamento adequado permitiu a sobrevida da paciente. Descrição do caso: Recém-nascido ( $\mathrm{RN}$ ) do sexo feminino com $2665 \mathrm{~g}$, nasceu com placas hiperceratósicas e fissuras difusas pelo corpo, ectrópio bilateral com oclusão palpebral, eclábio e pés e mãos fletidos características de Ictiose congênita do tipo arlequim. O acitretin foi iniciado aos 7 dias de vida associado ao tratamento suportivo, evitando o óbito neonatal. Comentários: Atribui-se a sobrevida da paciente à intervenção com acitretin oral precoce e os cuidados intensivos neonatais.

Desccritores: Ictiose lamelar; Anormalidades congênitas; Dermatologia; Pediatria.

\begin{abstract}
Harlequin Ichthyosis has a prevalence of 1/100.000 births and is frequent that the death occurs in neonatal period. Objective: to describe a case in which the adequate treatment allowed the patient's survival. Case description: Newborn, female, with $2665 \mathrm{~g}$, was born with hyperkeratotic plaques and diffuse fissures in the body, bilateral ectropion with palpebral occlusion, foot and hands flexed, characteristics of congenital harlequin Ichthyosis. Acitretin was started in the first 7 days associated with supportive treatment, preventing that the newborn evolved to neonatal death. Comments: The survival of the patient is attributed to the precocious intervention with acitretin and the intense care in the neonatal period.
\end{abstract}

Keywords: Ichthyosis, lamellar; Congenital abnormalities; Dermatology; Pediatrics.

1. Estudante de Medicina da Universidade Federal do Paraná, Curitiba, PR. ORCID: Teixeira IS - https://orcid.org/0000-0003-21359314; Rodrigues G - https://orcid.org/0000-0002-4001-5528; Nazar G - https://orcid.org/0000-0002-2227-4358; Neves IS - https:// orcid.org/0000-0002-3946-9784.Email: isabelle.sasso13@gmail.com,gre.kfrodrigues@gmail.com, Guilherme.nazar@gmail.com, izabellenneves@gmail.com.

2. Fellow em Dermatologia Pediátrica no Hospital de Clínicas da Universidade Federal do Paraná. Curitiba, Paraná, Brasil. Zanatta DA - https://orcid.org/0000-0002-4910-2441; daniellezanatta@gmail.com

3. Professora do Departamento de Pediatria, Serviço de Dermatologia Pediátrica do Hospital das Clínicas da Universidade Federal do Paraná. Curitiba, Paraná, Brasil. Carvalho VO https://orcid.org/0000-0002-4578-8781. Email: rcarvalho50@hotmail.com

Endereço para correspondência: Isabelle Sasso Teixeira. Hospital de Clínicas da Universidade Federal do Paraná. R. Gen. Carneiro, 181. Alto da Glória, Curitiba, PR. CEP: 80060-900. E-mail: isabelle.sasso13@gmail.com. 


\section{INTRODUÇÃO}

$\mathrm{N}$ as ictioses ocorrem alterações na queratinização cutânea, com gravidade variável. Têm caráter hereditário, entretanto, existem formas adquiridas, secundárias a doenças infecciosas, autoimunes, deficiência nutricional e doenças malignas ${ }^{1}$. As hereditárias são determinadas por mutações em genes que codificam proteínas estruturais ou enzimas envolvidas nas funções celulares, desde reparo do DNA até homeostase da barreira cutânea ${ }^{1}$. Anormalidades nestes componentes desencadeiam hiperplasia epitelial com excesso de estrato córneo acompanhado de descamação anormal ${ }^{2}$.

A forma arlequim apresenta incidência de 1/100.00 nascimentos e ocorre por mutação do gene $\mathrm{ABCA} 12^{3}$. A taxa de mortalidade durante o período neonatal é de aproximadamente $50 \%{ }^{4}$. As escamas são espessas lembrando uma armadura, envolvendo toda a pele, resultando em alterações na regulação térmica, no equilíbrio hidroeletrolítico e na proteção contra infecções, além de provocar dificuldade respiratória pela restrição física à expansão da caixa torácica. Após o nascimento, a criança desenvolve rachaduras profundas e fissuras associados a acentuados ectrópios, eclábio e malformações do pavilhão auricular ${ }^{5}$.

O objetivo deste relato de ictiose arlequim foi descrever o tratamento no período neonatal e a evolução aos três anos de vida.

\section{DESCRIÇÃO DO CASO}

Primigesta de 25 anos foi admitida em trabalho de parto com 36 semanas e 6 dias de gestação, e encaminhada para cesárea por distocia. Ultrassonografia com 28 semanas apontou anormalidades do feto, com hipoplasia de face, nariz curto, ponte nasal plana, hipoplasia maxilar e protrusão de língua. Pré-natal com diabetes mellitus gestacional controlado.

Recém-nascido do sexo feminino, pesou $2665 \mathrm{~g}$ ao nascimento com Apgar no $1^{\circ}$ e $5^{\circ}$ minuto de vida de 8 e 9 , respectivamente. Possuía placas hiperceratósicas e fissuras difusas pelo corpo (Figura 1), compatíveis com ictiose congênita do tipo arlequim, sendo admitida em UTI neonatal.

A clínica da paciente incluía ectrópio superior bilateral com oclusão palpebral - corrigido com dois anos de vida -, eclábio, pés e mãos com padrão flexor, hipoplasia bilateral de pavilhão auricular, com perda auditiva bilateral moderada do tipo condutiva.

Desde o segundo dia de vida, recebeu tratamento com múltiplos antimicrobianos, de acordo com as hemoculturas. Foi iniciado tratamento com acitretin aos 7 dias de vida, evoluindo com diminuição das fissuras e da descamação, com posterior aspecto de eritrodermia ictiosiforme congênita. $\mathrm{O}$ tratamento também incluiu uso contínuo de gel oftálmico com dexpantenol, colírio lubrificante, hidratantes corporais com petrolato, no período neonatal e, a partir dos 60 dias, $\mathrm{N}$-acetilcisteína $5 \%$ e ureia $5 \%$. Apresentou melhora significativa da descamação cutânea, sendo menos intensa em face e mais acentuada nos membros, estando atualmente com três anos de idade e bom controle da doença. (Figura 2).

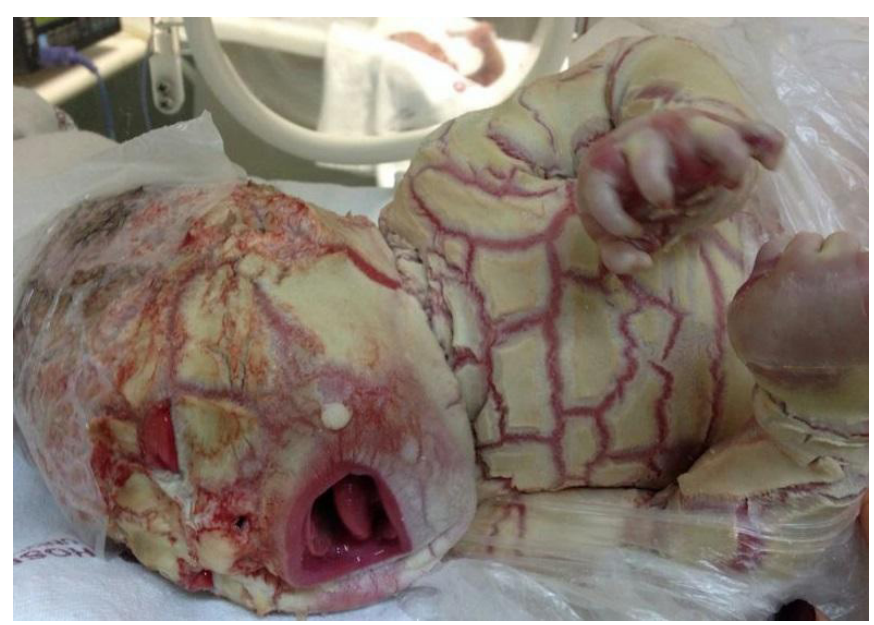

Figura 1: Escamas espessas, fissuras profundas e ectrópio com 2 horas de vida 

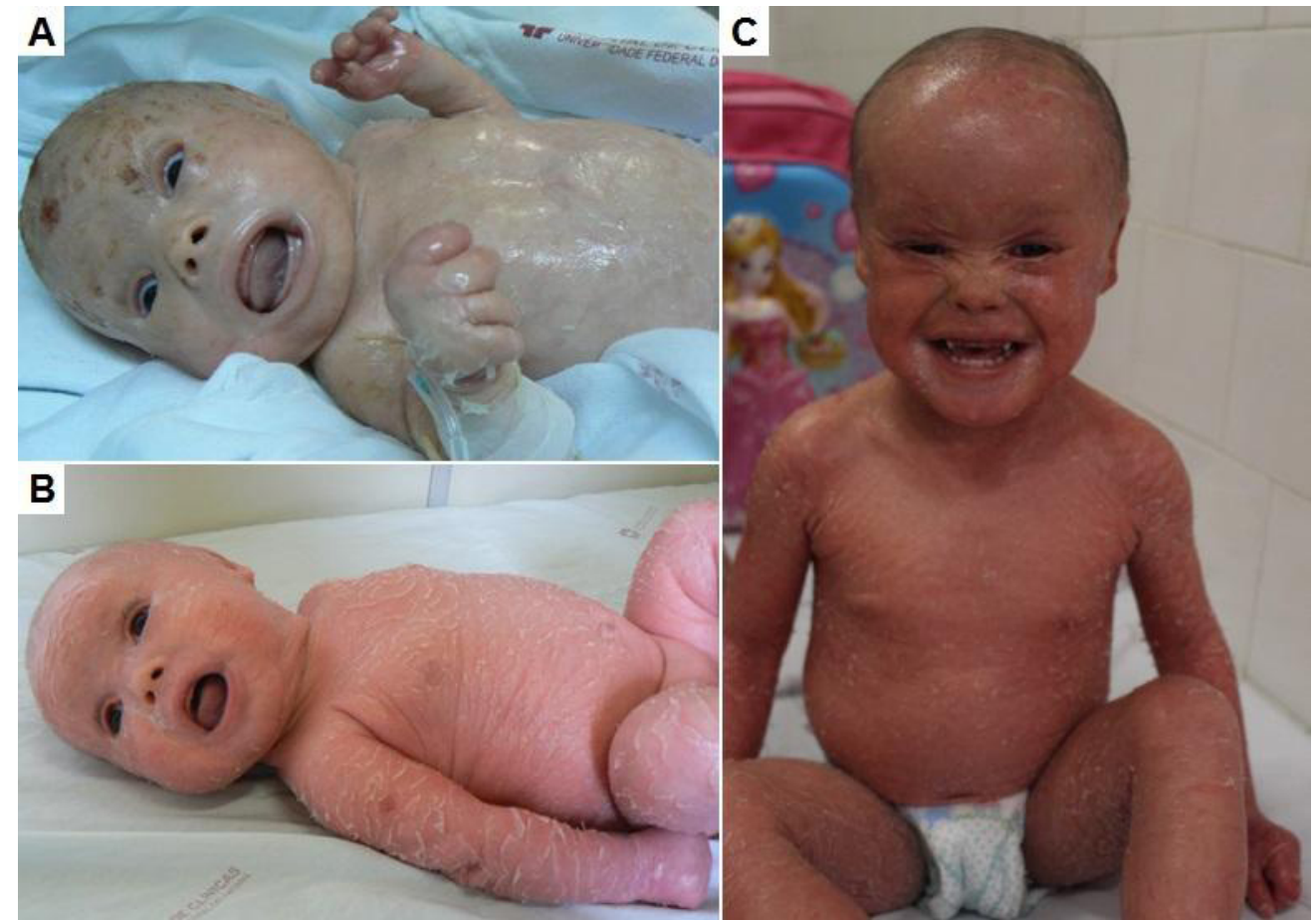

Figura 2: (A) - Após 30 dias do uso do acitretin com melhora importante, persiste com ectrópio e eclábio; (B) - eritema e descamação difusa aos 3 meses e (C) - os 3 anos

\section{DISCUSSÃO}

A ictiose arlequim foi associada a altas taxas de mortalidade e morbidade, contudo, com a introdução de tratamento precoce com retinóides via oral e com os cuidados em Unidade de Terapia Intensiva Neonatal, a taxa de mortalidade tornou-se menor do que $50 \% .^{6,7} \mathrm{O}$ uso de retinóides atua na prevenção de hiperceratose e na diminuição de escamas características da ictiose. ${ }^{8} \mathrm{O}$ acitretin é um análogo sintético do ácido retinóico que permite controlar a proliferação epitelial ${ }^{9}$.

Outro fator que influencia no prognóstico da doença é o diagnóstico pré-natal, realizado por meio da ultrassonografia durante o terceiro trimestre. Achados sugestivos são orelhas rudimentares, contratura em flexão nos joelhos, líquido amniótico com partículas densas, língua protuberante e lábios evertidos ${ }^{6,7}$. $\mathrm{Na}$ ecografia realizada durante a $28^{\mathrm{a}}$ semana de gestação, foram observadas anormalidades faciais, como malformações orais e língua protuberante. Entretanto, nesse momento, esses achados não foram correlacionados à ictiose, mas sim à malformação no desenvolvimento da cavidade oral. Em gestantes com história prévia de filhos com ictiose arlequim é possível realizar análise fetal do DNA do líquido amniótico ou da vilosidade coriônica em busca de mutações no gene ABCA12 10,11 .

A variante arlequim não tem cura e seu tratamento é suportivo, visando prevenir ou tratar complicações ${ }^{12}$. O suporte nutricional é necessário devido ao eclábio, que dificulta a amamentação ${ }^{12}$, na paciente aqui relatada foi possível alimentar via oral no segundo dia de vida. Está indicado o uso de petrolato, como a vaselina, e outros hidratantes tópicos a fim de reduzir perdas hídricas e eletrolíticas pela pele, contudo é comum que os pacientes recebam suporte intravenoso de fluídos ${ }^{13,14}$. Estes pacientes também realizam exames de cultura diariamente na primeira semana de vida e semanalmente durante o período de internamento, para o controle de infecções com o intuito de detectar precocemente a proliferação de bactérias e tratar precocemente sepse ${ }^{15}$, como observado na nossa paciente que evoluiu com episódios de sepse bacteriana e fúngica. O uso de colírios é necessário, pois o ectrópio dificulta a ação lubrificante lacrimal ${ }^{6}$.

A sobrevivência da paciente em questão provavelmente está relacionada ao tratamento com acitretin iniciado no final da primeira semana de vida, medida associada a aumento da sobrevida em $80 \%$, assim como ao manejo adequado das infecções ${ }^{6}$. Dessa forma, a minimização da perda transepidermica de água é uma das chaves para sobrevida nesta dermatose, pois previne desequilíbrio eletrolítico, desregulação de temperatura, estresse respiratório, desnutrição e infecções ${ }^{15}$.

Os fenótipos da ictiose podem mudar em resposta ao tratamento com retinóides. A criança com ictiose arlequim que sobrevive após o período neonatal, pode ter evolução similar a outro tipo de ictiose, a eritrodermia 
ictiosiforme congênita, uma forma mais branda da doença ${ }^{1}$. Tal evolução parece estar associada a mutações heterozigóticas do gene ABCA12 assim como a natureza de sua mutação e sua localização dentro da proteína ${ }^{6,17,18}$. Esse tipo tem características como eritema generalizado, anormalidades no couro cabeludo, alopécia, ${ }^{1}$ presentes na

\section{REFERÊNCIAS}

1. Oji V, Tadini G, Akiyama M, Bardon CB, Bodemer C, Bourrat, E, et al. Revised nomenclature and classification of inherited ichthyoses: results of the First Ichthyosis Consensus Conference in Soreze 2009. J Am Acad Dermatol. 2010; 63:607-41. doi: https://doi.org/10.1016/j.jaad.2009.11.020.

2. Schmuth M, Gruber R, Elias PM, Williams ML. Ichthyosis update: towards a function-driven model of pathogenesis of the disorders of cornification and the role of corneocyte proteins in these disorders. Adv Dermatolol. 2007;23:231-56. doi: https://doi.org/10.1016/j.yadr.2007.07.011.

3. Kelsell PD, Norgett EE, Unsworth H, Teh MT, Cullup $\mathrm{T}$, Mein CA, et al. Mutations in ABCA12 underlie the severe congenital skin disease harlequin ichthyosis. Am J Human Genet. 2005;76(5):794-803. doi: https://doi. org/10.1086/429844.

4. Harvey HB, Shaw MG, Morrell DS. Perinatal management of harlequin ichthyosis: a case report and literature review. J Perinatol. 2010;30(1):66. doi: https://doi.org/10.1038/ jp.2009.100.

5. Lai-Cheong JE, McGrath JA. Advances in understanding the genetic basis of inherited single gene skin barrier disorders: New clues to key genes that may be involved in the pathogenesis of atopic dermatitis. An Bras Dermatol. 2006;81(6):567-71. https://doi.org/10.1590/S0365-05962006000600009.

6. Rajpopat S, Moss C, Mellerio J, Vahlquist A, Gånemo A, Hellstrom-Pigg M, Kennedy C. Harlequin ichthyosis: a review of clinical and molecular findings in 45 cases. Arch Dermatol. 2011;147(6):681-6. doi: https://doi.org/10.1001/ archdermatol.2011.9.

7. Rathore S, David LS, Beck MM, Bindra MS, Arunachal G. Harlequin ichthyosis: prenatal diagnosis of a rare yet severe genetic dermatosis. J Clin Diagn Res. 2015:9(11):QD04. doi: https://doi.org/10.7860/JCDR/2015/15250.6705.

8. DiGiovanna JJ, Mauro T, Milstone LM, Schmuth M, Toro JR. Systemic retinoids in the management of ichthyoses and related skin types. Dermatol Ther. 2013;26(1):26-38. doi: https://doi.org/10.1111/j.1529-8019.2012.01527.x.

9. Gulasi S. Congenital ichthyosis: a case treated successfully with acitretin. Iran J Pediatr. 2016;26(5). doi: https://doi. org/10.5812/ijp.2442. paciente relatada.

Apesar da gravidade da doença, a ictiose arlequim pode ter evolução favorável, se houver diagnóstico e tratamento precoces. A continuação do uso dos retinóides deve ser feita pelo resto da vida, conforme a resposta terapêutica, assim como acompanhamento multidisciplinar para a redução da morbidade relacionada à doença.

10. Yanagi T, Akiyama M, Sakai K, Nagasaki A, Ozawa N, Kosaki R, et al. DNA-based prenatal exclusion of harlequin ichthyosis. J Am Acad Dermatol. 2008;58(4):653-56. doi: https://doi.org/10.1016/j.jaad.2007.12.018.

11. Ahmed H, O'toole EA. Recent advances in the genetics and management of harlequin ichthyosis. Pediatr Dermatol. 2014;31(5):539-46. doi: https://doi.org/10.1111/pde.12383.

12. Koochek A, Choate KA, Milstone LM. Harlequin ichthyosis: neonatal management and identification of a new ABCA12 mutation. Pediatric Dermatol. 2014;31(2):e63-e64. doi: https://doi.org/10.1111/pde.12263.

13. Edwards WH, Conner JM, Soll RF, Vermont Oxford Network Neonatal Skin Care Study Group. The effect of prophylactic ointment therapy on nosocomial sepsis rates and skin integrity in infants with birth weights of 501 to 1000g. Pediatrics. 2004;113(5):1195-203. doi: https://doi. org/10.1542/peds.113.5.1195.

14. Czarnowicki T, Malajian D, Khattri S, da Rosa JC, Dutt R, Finney R, et al. Petrolatum: barrier repair and antimicrobial responses underlying this "inert" moisturizer. J Allergy Clin Immunol. 2016;137(4):1091-102. doi: https://doi. org/10.1016/j.jaci.2015.08.013.

15. Glick JB, Craiglow BG, Choate KA, Kato H, Fleming RE, Siegfried E, Glick SA. Improved management of harlequin ichthyosis with advances in neonatal intensive care. Pediatrics. 2017;139(1):e20161003. doi: https://doi. org/10.1542/peds.2016-1003.

16. Tontchev G, Silverberg NB, Shlasko E, Henry C, Roberts JL, Roth MZ. Techniques for toddlers: linear band incision for harlequin ichthyosis with associated compartment syndrome. Pediatr Dermatol. 2014;31(5):625-9. doi: https:// doi.org/10.1111/pde.12446.

17. Lefèvre C, Audebert S, Jobard F, Bouadjar B, Lakhdar H, Boughdene-Stambouli $\mathrm{O}$, et al. Mutations in the transporter ABCA12 are associated with lamellar ichthyosis type 2 . Hum Mol Genet. 2003;12(18):2369-78. doi: https://doi. org $/ 10.1093 / \mathrm{hmg} / \mathrm{ddg} 235$.

18. Sakai K, Akiyama M, Yanagi T, et al. ABCA12 is a major causative gene for non-bullous congenital ichthyosiform erythroderma. J Invest Dermatol. 2009;129:2306-9. doi: https://doi.org/10.1038/jid.2009.23.

Submetido: 03.08.2019

Aceito: 16.07.2020 